\title{
Isolated Para-Aortic Nodal Recurrence in Endometrial Cancer
}

\author{
Motoki Matsuura*, Akimasa Takahashi, Hidetaka Nomura, Maki Matoda, Sanshiro Okamoto, \\ Hiroyuki Kanao, Kohei Omatsu, Kazuyoshi Kato, Kuniko Utsugi, Nobuhiro Takeshima
}

Department of Gynecologic Oncology, Cancer Institute Hospital, Tokyo, Japan

Email: *motoki.gyne@gmail.com

How to cite this paper: Matsuura, M., Takahashi, A., Nomura, H., Matoda, M., Okamoto, S., Kanao, H., Omatsu, K., Kato, K., Utsugi, K. and Takeshima, N. (2018) Isolated Para-Aortic Nodal Recurrence in Endometrial Cancer. Open Journal of $\mathrm{Ob}$ stetrics and Gynecology, 8, 669-675. https://doi.org/10.4236/ojog.2018.87071

Received: May 4, 2018

Accepted: June 24, 2018

Published: June 27, 2018

Copyright $\odot 2018$ by authors and Scientific Research Publishing Inc. This work is licensed under the Creative Commons Attribution International License (CC BY 4.0).

http://creativecommons.org/licenses/by/4.0/

\begin{abstract}
Aim: While cytoreductive surgery should be considered for patients with regional recurrence of endometrial cancer, the management of isolated para-aortic lymph node recurrence remains controversial. We investigated the frequency of isolated recurrence in the para-aortic lymph nodes and the effectiveness of surgery for such recurrence. Methods: A retrospectively maintained endometrial cancer database at the Cancer Institute Hospital in Japan was reviewed to identify sites of metastasis and recurrence. Results: A total of 2322 patients with endometrial cancer underwent primary treatment with systemic lymphadenectomy between 1984 and 2015. Systematic pelvic and para-aortic lymph node dissection was performed in 889 patients (Group 1), while 1433 patients underwent dissection of only the pelvic lymph nodes (Group 2). Although 16 patients (1.1\%) in Group 2 had isolated para-aortic recurrences, only 3 patients (0.3\%) in Group 1 had documented recurrences in the para-aortic lymph nodes $(\mathrm{p}=0.043)$. Although second recurrence occurred in $30.0 \%$ of patients who underwent surgical recurrence treatment, second recurrence occurred in $77.8 \%$ of patients who underwent non-surgical recurrence treatment $(\mathrm{p}=0.037)$. In addition, the overall survival rate for $\mathrm{pa}$ tients who underwent surgical recurrence treatment (80.0\%) was significantly higher than that for patients who underwent non-surgical recurrence treatment $(33.3 \%)(p=0.026)$. Conclusions: Patients who underwent dissection of only the pelvic lymph nodes had a higher frequency of isolated recurrence in the para-aortic lymph nodes. In addition, a relatively good prognosis could be achieved with surgical treatment for isolated recurrence in the para-aortic lymph nodes, which was better than that achieved using non-surgical methods.
\end{abstract}

\section{Keywords}

Endometrial Cancer, Recurrence, Isolated Para-Aortic Nodal Recurrence 


\section{Introduction}

In Japan, the incidence of endometrial cancer is increasing the fastest [1]. The reasons for this rising incidence include lifestyle changes among Japanese women such as diet, obesity, and age at pregnancy. Most endometrial cancer patients are diagnosed in the early stage and have a relatively good prognosis with 5-year survival rates at the Cancer Institute Hospital in Japan ranging from $97.5 \%$ in individuals at FIGO (International Federation of Gynecology and Obstetrics) stage I to $93.9 \%$ for those with stage II disease. However, patients presenting with FIGO stage III endometrial cancer (accounting for 10\% - 15\% of patients with endometrial cancer) have a poorer prognosis with a 5-year survival rate of 82.9\% at the Cancer Institute Hospital. In addition, the prognosis for recurrent endometrial cancer is poor, as a complete cure is very difficult. The sites of recurrence for endometrial cancer are the vagina, pelvis, lung, liver, and lymph nodes, including the para-aortic lymph nodes [2] [3] [4].

In endometrial cancer, recurrence usually occurs in multiple sites; therefore, chemotherapy or radiotherapy, rather than surgery, is recommended. While cytoreductive surgery should be considered for patients with regional recurrence, the management of isolated para-aortic lymph node recurrence remains controversial. No previous reports of cytoreductive surgery for isolated para-aortic lymph node recurrence exist. Therefore, we investigated the frequency of isolated recurrence in the para-aortic lymph nodes in a pelvic lymph node dissection group and a group of patients who underwent both pelvic and para-aortic lymph node dissection. In addition, we examined the effectiveness of surgery for isolated para-aortic lymph node recurrence.

\section{Methods}

Using a retrospectively maintained endometrial cancer database at the Cancer Institute Hospital in Japan, the records of all available data were reviewed to identify the sites of metastasis and recurrence. A total of 2322 patients with endometrial cancer underwent primary treatment with systemic lymphadenectomy between 1984 and 2015 at the Cancer Institute Hospital in Japan. During the study period, the management of endometrial cancer changed; therefore, some patients underwent dissection of only the pelvic lymph nodes while other patients underwent complete dissection of the pelvic and para-aortic lymph nodes. All patients underwent hysterectomy with bilateral salpingo-oophorectomy and underwent open surgery.

During the para-aortic lymph node dissection, nodal tissue on the right side was removed between the aorta medially and the right ureter laterally, extending from the right common iliac artery to the level of the insertion of the right ovarian vein into the vena cava. On the left side, nodal tissue was removed between the aorta medially and the left ureter laterally, extending from the common iliac artery to the level of the insertion of the left ovarian vein into the left renal vein.

Recurrence was diagnosed when a systemic enhanced-Computed Tomogra- 
phy (CT), Positron Emission Tomography (PET), or abdominal ultrasonography revealed metastatic tumors in the para-aortic area during the follow-up period. The consensus of multiple gynecologic oncologists determined the method of treatment for the recurrence according to the individual case; treatments included surgery (para-aortic lymphadenectomy), chemotherapy, and radiotherapy. Surgery wad selected for cases judged to be operable.

The overall survival rate was determined using the Kaplan-Meier method and survival curves were compared using the log-rank test. The chi-square test was used to evaluate the association between lymph node dissection and recurrence. A p-value was less than 0.05 was considered significant. Statistical analyses were performed using SPSS (Version 22, IBM, Japan).

\section{Results}

Systematic pelvic and para-aortic lymph node dissection was performed in 889 patients (Group 1) and 1433 patients underwent dissection of only the pelvic lymph nodes (Group 2).

In both groups, 19 patients $(0.8 \%)$ were diagnosed with isolated para-aortic recurrence. The clinicopathological characteristics of these 19 patients are shown in Table 1. Stratification by the risk of recurrence showed that all of these cases were of high (68.4\%) or intermediate risk (31.6\%).

Although 16 patients (1.1\%) in Group 2 had isolated para-aortic recurrence, only 3 patients $(0.3 \%)$ in Group 1 had documented recurrence in the para-aortic lymph nodes $(\mathrm{p}=0.043)$. The mean time to isolated para-aortic recurrence was 13 months (3 - 38 months). The distribution of the recurrent para-aortic lymph nodes is shown in Figure 1. The most frequent site of para-aortic node recurrence $(36.8 \%)$ was on the left side of the aorta between the level of the inferior mesenteric artery (IMA) and left renal vein. A total of 10 cases without pelvic lymph node metastasis in the initial surgery had recurrence in the para-aortic lymph nodes, among which, 4 patients were considered to be at high risk.

Recurrence was treated using surgical resection in 10 cases while 9 cases were treated with chemotherapy or radiotherapy alone. Although second recurrence occurred in $30.0 \%$ of patients in the surgery group, second recurrence occurred in $77.8 \%$ of those in the non-surgery group (i.e., chemotherapy or radiotherapy alone) ( $\mathrm{p}=0.037)$. Table 2 shows the relapse sites after undergoing recurrence treatment and Figure 2 shows the overall survival rate for both treatment groups. The overall survival rate of the patients who underwent surgical recurrence treatment $(80.0 \%)$ was significantly higher compared to that for the patients who underwent non-surgical recurrence treatment $(33.3 \%)(p=0.026)$. The overall survival rate for patients with isolated para-aortic recurrence was $55.6 \%$, equivalent to the prognosis for vaginal stump recurrence (51.2\%).

\section{Discussion}

The site of recurrence in endometrial cancer is diverse, with the method of 
Table 1. Clinical characteristics.

\begin{tabular}{cc}
\hline Age & $57.2(42-75)$ \\
Initial Sugery & 3 \\
TAH + BSO + PLA + PALA & 16 \\
TAH + BSO + PLA & \\
Histology & 11 \\
Endometrioid G1, G2 & 2 \\
Endometrioid G3 & 4 \\
Serous & 1 \\
Clear & 1 \\
Mixed & 13 \\
Risk assessment & 10 \\
High & 6 \\
Intermediate & 9 \\
Relapse treatment & \\
Surgery & \\
Non-surgery & \\
& \\
\hline
\end{tabular}

Table 2. Relapse after performing a recurrence treatment.

\begin{tabular}{ccc}
\hline & Surgery Group & Non-surgery Group \\
\hline None & 7 & 2 \\
Para-aortic lymphnode & 1 & 3 \\
Intrapelvic & 1 & 0 \\
Extrapelvic & 1 & 4 \\
\hline
\end{tabular}

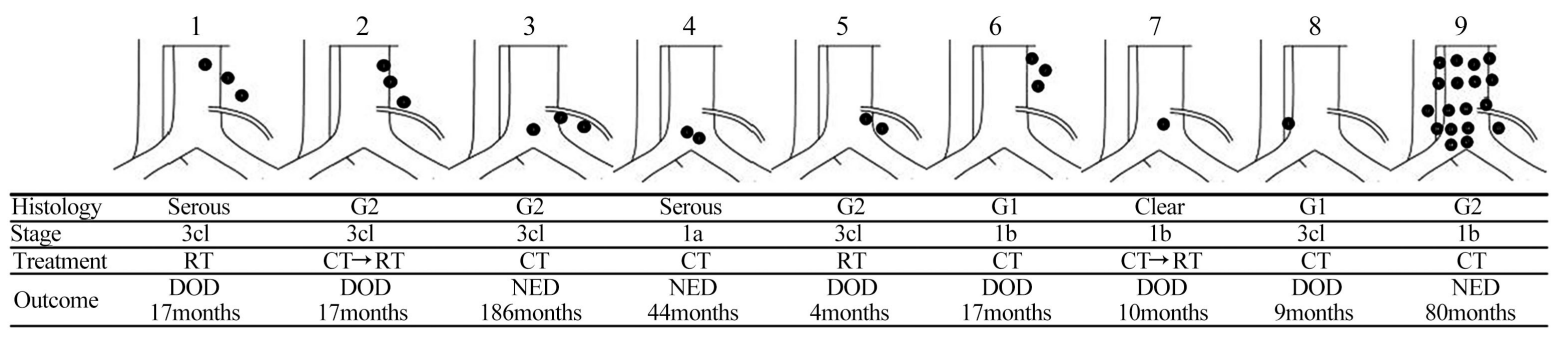

(a)

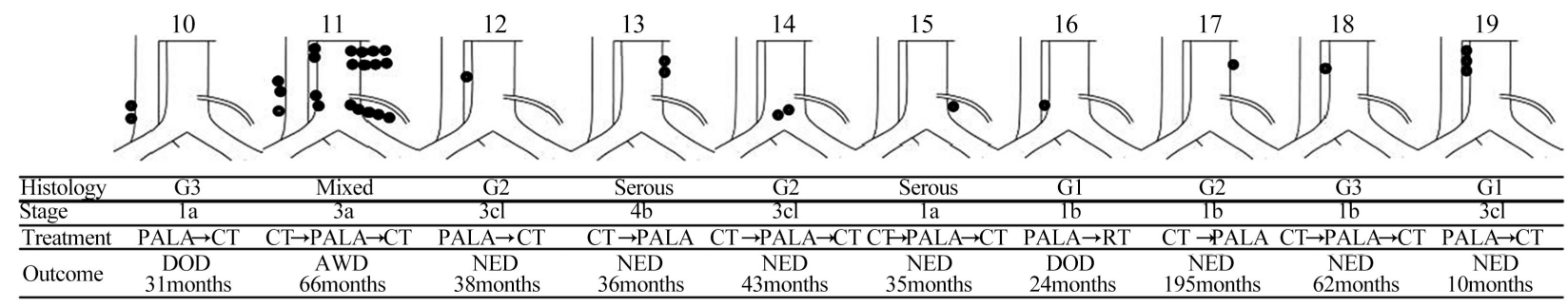

(b)

Figure 1. Lymph node distribution in 19 patients with isolated para-aortic recurrence. (a) The distribution in the non-surgery group ( $\mathrm{n}=9)$; (b) the distribution in the surgery group $(\mathrm{n}=10)$. NED, No evidence of disease; AWD, Alive with disease; DOD, Dead of disease; CT, Chemotherapy; RT, Radiotherapy; PALA, Pala-aortic lymphadenectomy; G1, Endometrioid grade 1; G2, Endometrioid grade 2; G3, Endometrioid grade 3. 


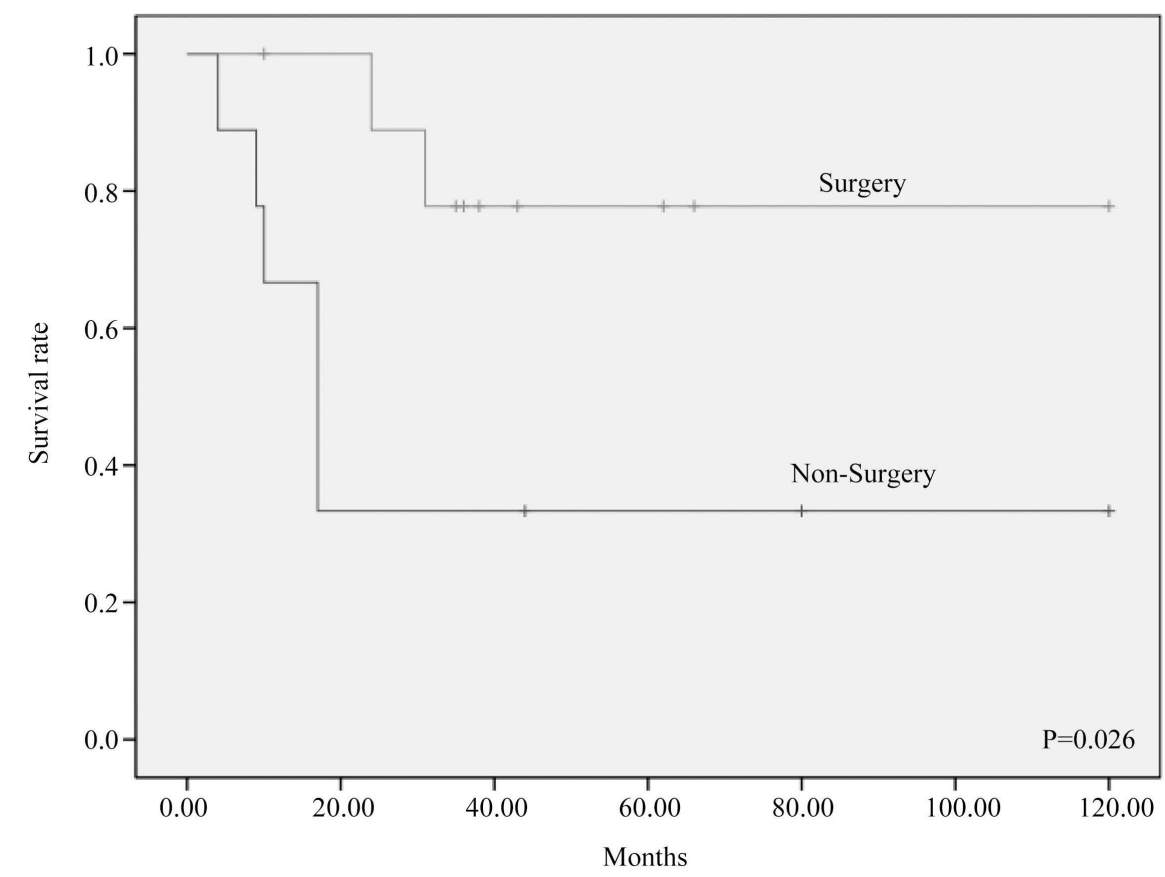

Figure 2. Overall survival rate for both groups. The overall survival rate was significantly higher in the surgery group compared to that for the non-surgery group $(\mathrm{p}=0.026)$.

treatment varying by the recurrence site. We found that the frequency of isolated recurrence in the para-aortic lymph nodes was significantly higher in patients who underwent dissection of only the pelvic lymph nodes during the initial surgery compared to those who underwent dissection of both the pelvic and para-aortic lymph nodes. In addition, the results suggest that for cases of recurrence in only the para-aortic lymph nodes, a relatively good prognosis could be achieved with surgical treatment, which was better than that achieved using chemotherapy or radiotherapy.

Rustum et al. reported the recurrent sites for 154 patients, which included the pelvis (34\%), abdomen (33\%), distant areas (27\%), and isolated para-aortic nodes (6\%) [2]. In contrast, the current study found that the frequency of isolated para-aortic lymph node recurrence among patients who underwent surgery with lymph node dissection was $0.8 \%$. The difference in the reported frequency may be related to the inclusion of cases without lymphadenectomy in the previous study. In addition, Rustum et al. reported that none of the patients with isolated para-aortic recurrence had grade 1 endometrioid adenocarcinoma [2]. In contrast, 4 patients with grade 1 endometrioid adenocarcinoma in the current study were diagnosed with para-aortic recurrence.

Although the prognostic value of lymphadenectomy has been well established, controversy exists regarding its therapeutic role [5]. Pelvic lymphadenectomy alone represents a half-way treatment. The para-aortic has been estimated to be involved in more than $50 \%$ of patients with lymphatic spread [5]. Todo et al. reported that approximately $70 \%$ of patients with stage $3 \mathrm{cl}$ disease had occult metastases in the para-aortic nodes detected at pathological ultra-staging [6]. 
Preventing isolated para-aortic lymph node recurrence is an important issue, even when para-aortic lymph node dissection was performed during the initial surgery. There were 3 cases in the present study with recurrence in the para-aortic lymph nodes, despite para-aortic lymph node dissection during the initial surgery. Moreover, patients who underwent dissection of only the pelvic lymph nodes had a higher frequency of isolated recurrence in the para-aortic lymph nodes compared to those who underwent pelvic and para-aortic dissection.

When a recurrence of endometrial cancer is diagnosed, the recurrence should be evaluated and identified as a local or regional recurrence, or as a systemic, disseminated recurrence. The number of relapse sites and time to relapse after the primary surgery are independent prognosticators for survival after relapse in patients with recurrent endometrial cancer [4]. If no other metastatic sites are detected, cytoreductive surgery should be considered. Cytoreductive surgery for recurrent endometrial cancer has been reported to have an optimal rate of 56\% $74 \%$, with a median survival period of 11.8 - 53.0 months for optimal cases [7] [8] [9] [10]. Elio et al. reported that the efficacy of a second cytoreduction was influenced negatively by a previous chemotherapy treatment and by the presence of carcinosis, whereas patients with a solitary recurrence had a greater possibility of achieving optimal debulking [8]. Barlin et al. reported that optimal cytoreduction was achievable in $52 \%-75 \%$ of patients with advanced or recurrent endometrial cancer and complete cytoreduction was possible in $18 \%-75 \%$ of cases [11].

No previous reports of cytoreductive surgery for isolated para-aortic lymph node recurrence exist. The overall survival for patients with recurrent endometrial cancer can be poor, and the recurrence site is the most important predictor of survival [3]. A good prognosis for those with an isolated recurrence may be achieved with surgery; therefore, isolated recurrence is considered one of the factors predicting a successful optimal surgery. The overall survival rate for patients with isolated para-aortic recurrence found in the current study (55.6\%) was decent and equivalent to the survival rate for vaginal stump recurrence (51.2\%).

A limitation of the current study is that selected patients underwent surgical recurrence treatment. These patients may represent a subgroup among patients with recurrent disease for whom surgery is more efficient at prolonging survival compared to chemotherapy and radiotherapy. There was a possibility that the state of the whole body was better in patients who selected surgery.

\section{Conclusion}

In conclusion, the present study revealed that the frequency of isolated recurrence in the para-aortic lymph nodes was significantly higher in patients who underwent dissection of only the pelvic lymph nodes compared to those who underwent pelvic and para-aortic dissection during the initial surgery. When the 
recurrence site is constrained to the para-aortic lymph nodes, the prognosis appears to be better with surgical treatment compared to that with non-surgical methods. Furthermore, the prognosis was relatively good for patients who underwent surgery for isolated recurrence in the para-aortic lymph nodes.

\section{Disclosure}

The authors have no conflicts of interest.

\section{References}

[1] Matsuura, M., Takahashi, A., Nomura, H., et al. (2018) Analysis of a Single Para-Aortic Lymph Node Metastasis in Endometrial Cancer. Journal of Cancer Science and Therapy, 10, 19-21. https://doi.org/10.4172/1948-5956.1000510

[2] Abu-Rustum, N.R., Chi, D.S., Leitao, M., et al. (2008) What Is the Incidence of Isolated Paraaortic Nodal Recurrence in Grade 1 Endometrial Carcinoma. Gynecologic Oncology, 111, 46-48. https://doi.org/10.1016/j.ygyno.2008.06.010

[3] Sartori, E., Laface, B., Gadducci, A., et al. (2003) Factors Influencing Survival in Endometrial Cancer Relapsing Patients: A Cooperation Task Force Study. International Journal of Gynecological Cancer, 13, 458-465. https://doi.org/10.1046/j.1525-1438.2003.13328.x

[4] Odagiri, T., Watari, H., Hosaka, M., et al. (2011) Multivariate Survival Analysis of the Patients with Recurrent Endometrial Cancer. Journal of Gynecologic Oncology, 22, 3-8. https://doi.org/10.3802/jgo.2011.22.1.3

[5] Bogani, G., Dowdy, S.C., Cliby, W.A., et al. (2014) Role of Pelvic and Para-Aortic Lymphadenectomy in Endometrial Cancer: Current Evidence. Journal of Obstetrics and Gynaecology Research, 40, 301-311. https://doi.org/10.1111/jog.12344

[6] Todo, Y., Suzuki, Y., Azuma, M., et al. (2012) Ultrastaging of Para-Aortic Lymph Nodes in Stage 3c1 Endometrial Cancer: A Preliminary Report. Gynecologic Oncology, 127, 532-537. https://doi.org/10.1016/j.ygyno.2012.08.026

[7] Scarabelli, C., Campagnutta, E., Giorda, G., et al. (1998) Maximal Cytoreductive Surgery as a Reasonable Therapeutic Alternative for Recurrent Endometrial Carcinoma. Gynecologic Oncology, 70, 90-93. https://doi.org/10.1006/gyno.1998.5017

[8] Campagnutta, E., Giorda, G., DePiero, G., et al. (2004) Surgical Treatment of Recurrent Endometrial Carcinoma. Cancer, 100, 89-96. https://doi.org/10.1002/cncr.11868

[9] Awtrey, C.S., Cadungog, M.G., Leitao, M.M., et al. (2006) Surgical Resection of Recurrent Endometrial Carcinoma. Gynecologic Oncology, 102, 480-488. https://doi.org/10.1016/j.ygyno.2006.01.007

[10] Bristow, R.E., Santillan, A., Zahurak, M.L., et al. (2006) Salvage Cytoreductive Surgery for Recurrent Endometrial Cancer. Gynecologic Oncology, 103, 281-287. https://doi.org/10.1016/j.ygyno.2006.03.011

[11] Barlin, J.N., Puri, I. and Bristow, R.E. (2010) Cytoreductive Surgery for Advanced or Recurrent Endometrial Cancer: A Meta-Analysis. Gynecologic Oncology, 118, 14-18. https://doi.org/10.1016/j.ygyno.2010.04.005 